Filaments and Their Environment 


\title{
Observations of Filament Structure and Dynamics
}

\author{
Oddbjørn Engvold \\ Institute of Theoretical Astrophysics, University of Oslo, \\ P.O. Box 1029, Blindern, N-0315 Oslo, Norway
}

\begin{abstract}
Past and recent observations of spatial and temporal variations in fine structure of solar prominences/filaments are reviewed. Magnetic fields permeate the prominence plasma and play a decisive role in shaping the fine structure and its dynamics. Consequently, the shape, orientation, and possibly the strength, of the magnetic field may be inferred from the observed sub-structures. The review discusses possible physical implications of small-scale structures in the low- $\beta$ prominence plasma.
\end{abstract}

\section{Introduction}

The fine structure of prominences holds the answers to fundamental questions in prominence research, such as how and why prominences form and how they are supported. The reasons for their fibril nature and varied complex forms are essentially unanswered at present. Magnetic fields permeate the prominence plasma and thereby serve to support the dense plasma against gravity and provide thermal insulation against the hot, surrounding corona.

The basic properties of solar prominences (filaments) are described and discussed in great details in proceedings and text books (cf., Jensen et al. 1979, Poland 1986, Ballester and Priest 1988, Priest 1989, Ruzdjak and TandbergHanssen 1990, and Tandberg-Hanssen 1995). It is the aim of the present review to discuss the physical significance of prominence structure, their dynamics, and relation to the environment.

One distinguishes between three basic types of filaments:

- Active Region Filaments, which form between and close to active region complexes.

- Quiescent Filaments that appear in or between weak remnants of active regions.

- Intermediate Filaments form between weak unipolar background field regions and active region complexes, and constitute a class in between to other two. 


\section{Spatial Structures}

Prominences, which are referred to as filaments when observed on the disk, always lie above the Polarity Inversion, i.e,. where the radial component of the network magnetic field changes sign. Quiescent filaments range in length from about 50,000 to $200,000 \mathrm{~km}$. They appear sometimes as continuous, elongated structures, but frequently also broken up into trains of separate segments. Prominences may appear as vertical, thin sheets, ranging in height from 10,000 $\mathrm{km}$ to $80,000 \mathrm{~km}$ above the photosphere.

Quiescent prominences consist of numerous thin threads, $5,000 \mathrm{~km}$ to 15,000 $\mathrm{km}$ long, and from $200 \mathrm{~km}$ to $1,500 \mathrm{~km}$ in width (Dunn 1960, Engvold 1976). In some prominences one also sees small, bright knots of similar dimensions. The thickness of the threads appears to increase with height above the solar limb (Engvold 1976). In filaments on the disk many threads are inclined to the direction of the long axes of the filament by about $30^{\circ}$.

At semi-regular intervals along a filament, the threads form pointed structures extending out from the filament body, which are referred to as barbs (cf., Kiepenheuer 1953). The ends of large filaments, which invariably bridge opposite magnetic polarities in the photosphere below, often fan out in the shape of a thin "shroud".

When seen at some distance from disk center, quiescent filaments display a well defined, sharp upper edge, which is labeled the filament spine. A spine often consists of several thin, long absorbing threads. At the limb spines are less conspicuous and generally more diffuse in $\mathrm{H} \alpha$. Images of prominences in HeII $304 \AA$ observed with EIT/SOHO invariably show much sharper upper edges, which suggests an increase of temperature with height.

\section{Filament Barbs and Inverse Polarity}

Rust and Kumar (1994) subscribe to the idea that filaments represent large, twisted flux ropes, where screw threads of the helix provide locations of magnetic support. If all filaments on the northern hemisphere were left-handed helical fields, and the magnetic fields were filled with $\mathrm{H} \alpha$ emitting gas, the observed field direction would be inverse. This model appears to disagree with the fact that barbs are not recognized as upward curved screw threads of a helix, and that barbs are never seen to cross the polarity inversion (Martin et al. 1994).

When filaments are situated close to the solar limb, barbs are usually seen only on the center-near side, which suggests that they are directed downwards. In $\mathrm{H} \alpha$ filter-grams from the solar limb, prominence barbs have the appearance of slightly curved pillars rooted in the chromosphere.

It has been noticed by Martin and co-workers that filament barbs seem to connect to the minority polarity flux on either side of the polarity inversion line (Martin 1994), whereas coronal arches appear to be rooted in the network flux (majority polarity). The preference for barbs to connect to the minority polarity of magnetic fields is evidently essential for the formation process and very existence of quiescent prominences. Engvold and Yi (1997) found that barbs of quiescent filaments connect to the weak field intra-cell region of the photospheric supergranulation cells, rather than to the much stronger $(\sim 1 \mathrm{kG})$ 
network field. Newly born barbs point into up-flow regions of the dynamic supergranulation cells, were possibly new weak magnetic flux emerges.

Martin et al. (1992) and Martin and Echols (1994) showed that barbs always bear to the right, when viewed from either side, in dextral filament channels, and to the left in sinistral channels. This latter relation is unambiguous, and one may therefore distinguish between dextral and sinistral filament channels simply from the "bearing" of the filament barbs.

\section{Flows and Oscillations}

The term quiescent is somewhat misleading in describing stable, large filaments, since they in reality are rather dynamic structures. One sees small-scale lineof-sight velocities of a few $\mathrm{km} \mathrm{s}^{-1}$, both in horizontal and vertical directions (Engvold 1972, Dunn 1960, Stellmacher and Wiehr 1994). Doppler measurements in disk filaments show both upwards and downwards mass motion (see Schmieder 1989, and papers cited therein). It was later noted by Yi et al. (1991) that the dominant vertical motion in the main body of filaments was oscillatory. Periodic velocities and intensity oscillations have a large range of periodicities, from less than $1 \mathrm{~min}$ to hours (Yi et al. 1991, Thompson and Schmieder 1991, Balthasar and Wiehr 1994). An excellent review of oscillations in prominence fine-structure is given by Joardar et al. (1997), who also discuss and classify hydromagnetic modes of oscillations in thin magnetic strings, which appear to be in good agreement with observations.

Unpublished observations in $\mathrm{H} \alpha$ by Jack Zirker and collaborators seem to show flow-type motions in barbs. The true sign of these flows is partly disturbed and confused by small-scale structures and motions in the chromosphere below.

\section{The Nature of Fine-Structure Threads}

It is generally assumed that the fine-structure threads outline their magnetic flux tubes. This view is substantiated by the fact that the threads of large, quiescent filaments are inclined relative to the filament long axis, similar to the magnetic field (Leroy 1989, Bommier et al. 1994, Bommier and Leroy 1998, these proceedings). On the other hand, one would not expect to find strongly inhomogeneous magnetic fields in low- $\beta$ filament plasmas. Therefore, one is led to to speculate whether the fine threads seen in $\mathrm{H} \alpha$ could represent "veins" of dense plasma in a relatively uniform magnetic field, rather than individual flux ropes? Such a model is, however, at variance with a notable fluctuation in the line-of-sight velocity of neighboring threads (Engvold 1972, Zirker and Koutchmy 1990, 1991, Stellmacher and Wiehr 1994), which seems to imply that the threads represent separate flux tubes. A possible weak interaction between neighboring threads is indicated by the findings of Mein and Mein (1991) that the velocity threads seem to be slightly thicker than the density threads.

In hedgerow prominences the lifetime of vertical threads is $\leq 10 \mathrm{~min}$ (Engvold 1976). High resolution $\mathrm{H} \alpha$ filtergrams obtained with the SVST, La Palma, show individual, thin filament threads appearing and disappearing in the course of one minute. This suggests that prominence plasma may be undergoing rapid 
variations in temperature and/or density. Such short lifetimes could imply that the prominence plasma is not in a stationary radiative state (Engvold 1980).

\section{Temperature Structure}

The core temperatures and electron densities of prominence plasma are, respectively, $\mathrm{T}_{e}=6000-8000 \mathrm{~K}$ and $\mathrm{N}_{e}=10^{10}-10^{11} \mathrm{~cm}^{-3}$ (Hirayama 1990). There is evidence of more low temperature gas in the prominence and cavity regions than one detects with "normal" H $\alpha$ exposure times. Leroy (1972) found signs of very faint $\mathrm{H} \alpha$ emission in the corona close to prominences. The same data was reexamined by Dermendjiev et al. (1994) who saw faint coronal $\mathrm{H} \alpha$ emission also in the form of long systems of drops and fibrils outlining large, smooth curves that one normally associates with magnetic loops. It was concluded from these studies that coronal cavities also contain a noticeable amount of low temperature gas. Furthermore, this view is supported by the observations of radio-filaments $(\lambda \geq 3 \mathrm{~cm})$ showing more cold filament material than $\mathrm{H} \alpha$ (Gopalswamy et al. 1991). The lower gas pressure in such regions must evidently be balanced by a higher magnetic pressure.

The fact that solar prominences contain gas at all temperatures between $10^{4}$ $\mathrm{K}$ and $10^{6} \mathrm{~K}$ is demonstrated by their strong EUV line emissions (KjeldsethMoe et al. 1979, de Boer et al. 1998, these proceedings). Pojoga (1994) studied the variation with height of the Emission Measure (EM) from EUV lines and concluded that the emitting structures have sub-resolution geometries. The nature of these geometries is still unknown. The following are two alternative scenarios:

1. The threads, situated in a hot coronal cavity with cold cores, are "wrapped" in a thin transition region perpendicular to the magnetic field (Chiuderi-Drago et al. 1992).

2. The individual threads are essentially isothermal, but a given thread may attain temperatures between $10^{4} \mathrm{~K}$ and $10^{6} \mathrm{~K}$ (Pojoga 1993).

Since magnetic flux ropes constituting filament threads must be anchored somewhere on either side of the filament, the ropes are evidently a lot longer than their observable lengths in $\mathrm{H} \alpha$. The observational results from wave motions in filament threads (Yi et al. 1991, Yi and Engvold 1991) seem to support this view. These results suggest that temperature and/or density vary along a given flux rope.

\section{The Paradox of Vertical Magnetic Threads}

Zirker et al. (1994) confirmed the results from earlier studies showing no evidence for "free fall" velocities in large, quiescent prominences where a vertical thread structure is well developed. The nagging question then becomes, if the threads are nearly vertical magnetic flux tubes, what prevents the gas from falling freely in the field of gravity? 
Fontenla et al. (1996) studied stationary prominence thread models including ambipolar diffusion and horizontal magnetic fields. However, such models fail to explain the structural character and dynamic aspects of threads.

Jensen (1990) suggested that non-linear Alfvén waves might provide support of prominence plasma. A similar idea was put forward by de Pontieu and Haerendel (1994) who claimed that weakly-damped Alfvén waves could explain the acceleration of spicules. However, wave support does not work for prominences, for the following reason: If wave motion shall influence the pressure beyond the normal thermal gas pressure, the wave amplitude $v$ must be:

$$
v>\left(\frac{2 k T}{\mu m_{H}}\right)^{\frac{1}{2}}
$$

In the case of prominence plasma with $\mathrm{T} \approx 8000 \mathrm{~K}$, the "equivalent" wave amplitude corresponds to $9.1 \mathrm{~km} \mathrm{~s}^{-1}$. Therefore, in order to increase the pressure scale height by a factor 100 , e.g. from $170 \mathrm{~km}$ to $17,000 \mathrm{~km}$, the wave amplitude must be $v \geq 90 \mathrm{~km} \mathrm{~s}^{-1}$. Such supersonic velocities are hardly ever observed in quiescent prominences.

Table 1. Estimates of radiative pressure exerted by absorption of some strong chromospheric lines. The values of $d \ell_{s}$ given in the 5th column are the maximum column heights that can be supported by the radiative pressure for a given line when $\rho=7 \times 10^{-14}\left[\mathrm{~g} \mathrm{~cm}^{-3}\right] . d \ell_{\tau \approx 1}$ is the distance in the gas element corresponding to an optical depth $\tau=1$.

\begin{tabular}{|l|c|c|c|c|r|}
\hline Transition & $F_{\lambda}{ }^{(*)}$ & $\begin{array}{c}\Delta \lambda \\
{[\AA]}\end{array}$ & $\begin{array}{c}\rho d \ell \\
{\left[\mathrm{g} \mathrm{cm}^{-2}\right]}\end{array}$ & $\begin{array}{r}d \ell_{s} \\
{[\mathrm{~km}]}\end{array}$ & $\begin{array}{r}d \ell_{\tau=1} \\
{[\mathrm{~km}]}\end{array}$ \\
\hline \hline Ly $\alpha$ & $2.0 \times 10^{4}$ & 1.5 & $1.2 \times 10^{-10}$ & 0.017 & 0.005 \\
Ly-C (912) & $2.0 \times 10^{4}$ & $\sim 120$ & $<9.3 \times 10^{-9}$ & $<1.30$ & 53 \\
H $\alpha$ & $1.5 \times 10^{5}$ & 0.60 & $1.2 \times 10^{-9}$ & 0.170 & 610 \\
Ba-C (3647) & $3.2 \times 10^{6}$ & $\sim 500$ & $<6.1 \times 10^{-6}$ & $<870$ & $4 \times 10^{7}$ \\
He I D & $3.0 \times 10^{6}$ & 0.35 & $4.0 \times 10^{-9}$ & 0.570 & 900 \\
He I 10830 & $1.0 \times 10^{6}$ & 0.75 & $2.9 \times 10^{-9}$ & 0.410 & 600 \\
Ca II H & $2.4 \times 10^{5}$ & 0.12 & $1.1 \times 10^{-10}$ & 0.160 & 150 \\
Ca II K & $2.4 \times 10^{5}$ & 0.14 & $1.3 \times 10^{-10}$ & 0.180 & 76 \\
\hline
\end{tabular}

(*) Units: [erg $\mathrm{cm}^{2} \mathrm{~s}^{-1} \mathrm{sr}^{-1} \AA^{-1}$ ]

The pressure exerted by incident photospheric and chromospheric radiation will balance a prominence element of column mass $\rho d \ell$ when:

$$
\rho d \ell=\frac{\pi F_{\lambda} \Delta \lambda}{g_{\odot} c}
$$

where $F_{\lambda}$ is the mean monochromatic flux over the spectral width $\Delta \lambda, g_{\odot}$ is the acceleration of gravity, $c$ is the speed of light, and $\rho$ is gas density. For the sake of argument we may here assume a structure thickness $d \ell \simeq 100 \mathrm{~km}$, which is the order of magnitude of the finest observed structures. A plasma density $\rho \simeq 7 \times 10^{-14}\left[\mathrm{~g} \mathrm{~cm}^{-3}\right]$ (Hirayama 1990) then yields a column mass 
$\rho d \ell \simeq 7 \times 10^{-7}\left[\mathrm{~g} \mathrm{~cm}^{-2}\right]$. It is important to note that radiative pressure provides support in the field of gravity for only an outer layer of thickness $\tau \simeq 1$. When an element is optically thin, only a correspondingly smaller fraction of the radiation will be absorbed and contribute to a radiative lift. Adopting incident radiative fluxes from Pierce and Allen (1977) and Vidal-Madjar (1977), and line absorption coefficients from Allen (1973), one finds (Table 1) that absorption in prominent lines of $\mathrm{H}, \mathrm{He}$ and $\mathrm{Ca}$ can support less than $1 \%$ of this column mass. Prominence plasma is transparent to the vastly greater energy in the incident continuum radiation, which provides even less radiative push. Considering that this example was calculated for a very small column mass, we may safely conclude that radiation pressure cannot support prominences.

A possible solution to the paradox of vertical magnetic threads could be that a thread of thickness, for instance, $300 \mathrm{~km}$ is actually a "rope" of a many much thinner fibers, which each undergoes rapid changes in temperature. In a given fiber, newly condensed $\mathrm{H} \alpha$ emitting gas is rapidly heated again to high temperature, before it attains a free falling velocity much larger than a few $\mathrm{km}$ $\mathrm{s}^{-1}$. The observable velocities along a resolved thread will be the collective result from many unresolved fibers in a thread. A more detailed study of such models is under way.

\section{Associated Structures}

\subsection{Bright rims}

Bright rims are often noticed in $\mathrm{H} \alpha$ filtergrams at the center-near side of filaments (Royds 1920, d'Azambuja and d'Azambuja 1948). Kostik and Orlova (1975) proposed that the brightening was the result of back scattering from the bottom parts of a filament to the chromosphere below. Some observers claim that the brightening is located in the lower edge of the filament. Heinzel et al. (1995) found that bright rims could result from diffusion of incident $\mathrm{H} \alpha$ radiation in the lower part of the filament. Paletou (1997) repeated these calculations in a 2-D geometry and concluded that radiative diffusion would be rather evenly distributed over the filament, and that the mechanism could not explain bright rims. Hence, the origin of filament bright rims is still not well understood.

\subsection{Cavities}

Eclipse observations have shown that quiescent prominences are embedded in a complex coronal structure of a darker cavity within arches at the base of thin coronal streamers (Saito and Tandberg-Hanssen 1973). The outer helmet streamer structure is often seen in white-light eclipse observations.

A unique set of high resolution, white-light images of the solar corona were obtained with the $3.6 \mathrm{~m}$ Canada-France-Hawaii Telescope on Mauna Kea during the eclipse of July 11, 1991 (Koutchmy et al. 1994, November and Koutchmy 1996). The images show arcades of concentric, fine-scale, dark and bright looplike structures extending above a small prominence at the limb. A small coronal cavity close to the prominence contains numerous fine, dark threads which demonstrate better than any data before that a cavity region is highly structured and inhomogeneous. 


\subsection{Bright X-ray axial structures}

Martin and McAllister (1995) studied the orientation of X-ray arcades over filament channels from Yohkoh data, and noticed a change in magnetic shear with altitude. The inferred change in direction of the magnetic field, from the low temperature up into the coronal arches above, is difficult to verify observationally. In Yohkoh images one occasionally sees transient, thin filamentary structures that run close to $\mathrm{H} \alpha$ filaments (Solberg and McAllister 1998, these proceedings). These X-ray filaments are, conceivably, associated with a layer of sheared magnetic fields, at some height in the cavity, where the magnetic spine of the filament touches the lower boundary of the helmet structure.

Acknowledgments. This author is indebted to T. Forbes, V. Gaizauskas, K. Harvey, S. F. Martin, E. Priest, A. van Ballegooijen, and J. Zirker for stimulating discussions and interactions in studies of solar filaments and prominences. This collaboration has been possible partly by the NSF Grant ATMM to Helio Research, and to NATO Grant No. 940116. Thanks also to Egil Leer for discussions on wave support. Sara Martin provided valuable comments to improve the manuscript.

\section{References}

Allen C W. 1973, Astrophysical Quantities, Univ. of London, The Athlon Press Ballester J L and Priest E R (eds.) 1988, Workshop on Dynamics and Structure of Solar Prominences, Universite de les Illes Baleares

Balthasar $\mathrm{H}$ and Wiehr E. 1994, A\&A, 286, 639

Bommier V, Landi Degl'Innocenti E, Leroy J-L, and Sahal-Bréchot S. 1994, Solar Phys., 154, 231

Chiuderi Drago F, Engvold O, and Jensen E. 1992, Solar Phys., 139, 47

d'Azambuja L and d'Azambuja M. 1948, Ann. Obs. Paris-Meudon, VI, 7

Dermendjiev V N, Mouradian Z, Duchlev P, and Leroy J-L. 1994, Solar Phys., 149,267

de Pontieu and Haerendel G. 1994, in Solar Coronal Structures, V. Rusin et al. (eds.), VEDA, Slovak Rep., p. 323

Dunn R B. 1960, PhD Thesis, Harvard University

Engvold O 1972, Solar Phys., 23, 346

Engvold O 1976, Solar Phys., 49, 283

Engvold O 1980, Solar Phys., 67, 351

Engvold O and Yi Z. 1997, in preparation

Fontenela J M, Rovira M, Vial J-P, and Gouttebroze P. 1996, ApJ, 466, 496

Gopalswamy N, White S M, and Kundu M R. 1991, ApJ, 379, 366

Heinzel P, Kotrc P, Mouradian, Z, and Buykliev G T. 1995, Solar Phys., 160, 19

Hirayama T. 1990, in Dynamics of Quiescent Prominences, (eds.) V. Ruzdjak and E. Tandberg-Hanssen, Lecture Notes in Physics 363, Springer-Verlag, New York, p. 187 
Jensen E, Maltby P, and Orrall, F Q (eds.) 1979, Physics of Solar Prominences, IAU Coll. 44, Oslo

Jensen E. 1990, in Dynamics of Quiescent Prominences, (eds.) V. Ruzdjak and E. Tandberg-Hanssen, Lecture Notes in Physics, 363, Springer-Verlag, New York, p. 129

Joardar P S, Nakariakov V M, and Roberts B. 1997, Solar Phys., 173, 81

Kiepenheuer, K O 1953, in The Sun, G. P. Kuiper (ed.), Univ. of Chicago Press, Chicago, p. 395

Kjeldseth-Moe O, Cook J W, and Mango S A. 1979, Solar Phys., 61, 319

Kostik R I and Orlova T V. 1975, Solar Phys., 45, 119

Koutchmy S et al. 1994, A\&A, 281, 249

Leroy J-L. 1972, Solar Phys., 25, 413

Leroy J-L. 1989, in Dynamics and Structure of Quiescent Solar Prominences,

E.R. Priest (ed.), Kluwer Acad. Publ., Dordrecht, Holland, p. 77

Martin S F. 1994, BAAS, 26, 1522

Martin S F, Marquette, W H, and Bilimoria R 1992, in The Solar Cycle, K. L. Harvey (ed.), ASP Conf. Ser., Vol. 27, San Francisco, p. 53

Martin S F, Bilimoria R and Tracadas P W. 1994, in Solar Surface Magnetism, (eds.) R J Rutten and C J Schrijver, Kluwer Acad. Publ., Dordrecht, Holland, p. 303

Martin S F and Echols C R. 1994, in Solar Surface Magnetism, (eds.) R J Rutten and C J Schrijver, Kluwer Acad. Publ., Dordrecht, Holland, p. 339

Martin S F and McAllister A H. 1995, BAAS, 27, 961

Mein P and Mein N. 1991, Solar Phys., 136, 317

November L J and Koutchmy S. 1996, ApJ, 466, 512

Paletou F. 1997, A\&A, 317, 244

Pierce A K and Allen R G. 1977, The Solar Output and Its Variations, Colorado Associated University Press, Boulder, O.R. White (ed.), p. 169

Pojoga S. 1994, in Solar Coronal Structures, (eds.) V Rusin, P Heinzel and J-C Vial, VEDA Publ. Company, Slovak Rep., p. 357

Poland A I (ed.), 1986, Coronal and Prominence Plasmas, NASA Conference Publication 2442, Washington, DC

Priest E R (ed.), 1989, Dynamics and Structure of Quiescent Solar Prominences,

Kluwer Acad. Publ., Dordrecht, Holland

Royds T. 1920, Kodaikanal Obs. Bull., 63, 289

Rust D M and Kumar A. 1994, Solar Phys., 155, 69

Ruzdjak V and Tandberg-Hanssen E (eds.) 1990, Lecture Notes in Physics 363, Springer-Verlag, New York

Saito K and Tandberg-Hanssen E. 1973, Solar Phys., 31, 105

Schmieder B. 1989, in Dynamics and Structure of Quiescent Solar Prominences,

E R Priest (ed.), Kluwer Acad. Publ., Dordrecht, Holland, p. 15

Stellmacher G and Wiehr E. 1994, A\&A, 290, 655 
Tandberg-Hanssen E. 1995, The Nature of Solar Promiences, Kluwer Acad. Publ., Dordrecht, Holland

Thompson W T and Schmieder B. 1991, A\&A, 243, 501

Vidal-Madjar A. 1977, The Solar Output and Its Variations, Colorado Associated University Press, Boulder, O.R.White (ed.), p. 213

Yi Z, Engvold O, and Keil S L. 1991, Solar Phys., 132, 63

Yi Z, Engvold O. 1991, Solar Phys., 134, 275

Zirker J B and Koutchmy S. 1990, Solar Phys., 127, 109

Zirker J B and Koutchmy S. 1991, Solar Phys., 131, 107

Zirker J B, Engvold O, and Yi Z. 1994, Solar Phys., 150, 81 